\title{
RELAÇÃO DE POLIMORFISMOS NOS GENES ADIPOQ, TMEM18, FNDC5 E CONCENTRAÇÕES SÉRICAS DE ADIPONECTINA E IRISINA COM AUMENTO DE PARÂMETROS DE ADIPOSIDADE EM CRIANÇAS E ADOLESCENTES
}

\author{
NEUMANN, I. ${ }^{1}$, MATOS, W.L. ${ }^{1}$, GOUVEIA, A.M.A. ${ }^{2}$, REUTER, C.P. ${ }^{3}$, TODENDI, P.F. ${ }^{4}$, VALIM, M.R.A. 5 \\ PALAVRAS-CHAVE: Obesidade. SNP. TMEM18. ADIPOQ. FNDC5. Escolares.
}

\begin{abstract}
RESUMO
Índices globais de obesidade aumentaram exponencialmente nas últimas décadas. Sendo assim, essa condição tornou-se motivo de preocupação na saúde pública. Além de fatores ambientais, a genética tem contribuído para essa situação. SNPs nos genes ADIPOQ, TMEM18 e FNDC5, bem como concentrações séricas de adiponectina e irisina têm sido associadas com aumento de parâmetros de adiposidade. A partir disso, o objetivo do estudo foi relacionar a presença dos polimorfismos rs182052 no ADIPOQ, rs16835198 no FNDC5, rs6548238 no TMEM18 e níveis plasmáticos de adiponectina e irisina com parâmetros antropométricos e bioquímicos em escolares da região de Santa Cruz do sul. Estudo de caráter transversal, contou com a participação de 500 escolares, com idades entre 7 e 17 anos. A genotipagem dos SNPs foi realizada pelo qPCR. 0 tratamento dos dados deu-se por estatística descritiva, UNIANOVA ajustado por sexo e idade e Kruskal-Wallis. Portadores dos genótipos AG+AA para o polimorfismo rs182052 no ADIPOQ foram associados com maiores médias para circunferência da cintura $(p=0,042)$ e triglicerídeos $(p=0,021)$. Indivíduos eutróficos com os genótipos GT e GG para o SNP rs16835198 no FNDC5 foram associados com maiores valores de IMC $(p=0,033)$ e circunferência da Cintura $(p=0,044)$. Escolares com excesso de peso portadores do genótipo CC para o polimorfismo rs6548238 no TMEM18 estiveram relacionados a maiores médias para colesterol total $(p=0,021)$. Escolares obesos ou com sobrepeso apresentaram maiores médias para as variáveis: circunferência da cintura, percentual de gordura, pressão arterial sistólica e diastólica, c-LDL e triglicerídeos. Os polimorfismos rs182052 no ADIPOQ, rs16835198 no FNDC5 e rs6548238 no TMEM18 demonstraram associação com as variáveis estudadas. Níveis séricos de adiponectina e irisina não foram relacionados com os parâmetros investigados
\end{abstract}

\section{RELATION OF POLYMORPHYSMS IN THE ADIPOQ, FNDC5, TMEM18 AND SERUM CONCENTRARION OF ADIPONECTIN AND IRISIN WITH HIGHER ADIPOSITY PARAMETERS IN CHILDREN AND ADOLESCENTS. ABSTRACT}

\author{
KEYWORDS: Obesity. SNP. TMEM18. ADIPOQ. FNDC5. Schoolchildren.
}

\begin{abstract}
Overall obesity rates have increased exponentially in the last decades. Therefore, this condition has become a concern in public health. In addition to environmental factors, genetics contributed to this situation. SNPS in ADIPOQ, TMEM18, FNDC5 and serum levels of adiponectin and irisin have been associated with increased adiposity parameters. From this, the objective of the study was to relate to the presence of polymorphisms rs182052 in ADIPOQ, rs16835198 in FNDC5, rs6548238 in TMEM18 and plasmatic levels of adiponectin an irisin with anthropometric and biochemical parameters. A cross-sectional study was attended by 500 schoolchildren, aged between 7 and 17 years. Genotyping of SNPs was performed by qPCR. The data treatment was by descriptive statistics, UNIANOVA adjusted by sex and age and Kruskal-Wallis. Preschoolchildren obese or overweight with the AG+AA genotype for rs182052 in ADIPOQ polymorphism were associated with higher averages for waist circumference $(p=0,042)$ and triglycerides $(p=0,021)$. Eutrophic individuals with GT and GG genotypes for the SNP rs16835198 in FNDC5 were associated with higher BMI ( $p=$ $0,033)$ and waist circumference $(p=0,044)$. Overweight school children carrying the CC genotype for rs6548238 polymorphism in TMEM18 were related to higher mean for total cholesterol $(p=0,021)$. Obese or overweight schoolchildren presented higher averages in waist circumference, percentage of fat, systolic and diastolic blood pressure, LDL-c and triglycerides. Polymorphisms rs182052 in ADIPOQ, rs16835198 in FNDC5 and rs6548238 in TMEM18 demonstrated association with the variables studied. Serum levels of adiponectin and irisin were not related to the investigated parameters.
\end{abstract}




\section{INTRODUÇÃO}

Índices globais de obesidade aumentaram exponencialmente nas últimas décadas (KINLEN et al., 2017). O número de meninas nessa condição aumentou de 5 milhões em 1975 para 50 milhões em 2016; já para os meninos, nesse mesmo período, aumentou de 6 milhões para 74 milhões (NCD-RisC, 2017). Nesse contexto epidêmico, a obesidade tornou-se motivo de preocupação em saúde pública (ARROYO-JONHSON e MINCEY, 2016).

Mudanças nos fatores ambientais como aumento da disponibilidade de alimentos com alto teor calórico, marketing alimentício, questões socioeconômicas e a baixa atividade física da população foram determinantes para o aumento nos índices de obesidade (SWINBURN et al., 2011). Além disso, características genéticas, como os SNPs, (Single Nucleotide Polimorfism), que são uma variação de nucleotídeo único, têm sido associados ao aumento de parâmetros de adiposidade na população (LEÓN-MIMILA et al., 2013).

Níveis séricos das proteínas adiponectina e irisina, que são codificadas pelos genes ADIPOQ e FNDC5, respectivamente, têm sido associados a um menor risco de desenvolver doenças cardiovasculares (KHYROU et al., 2017), aumento da sensibilidade a insulina (YADAV et al., 2012) e escurecimento do tecido adiposo (BOSTROM et al., 2012). Enquanto isso, a presença de SNPs em genes específicos, como o rs182052 no ADIPOQ, rs16835198 no FNDC5 e rs6548238 TMEM18 têm sido associados com alterações metabólicas específicas, dentre elas, diabetes do tipo 2 e dislipidemia (KHIDIR et al., 2017; LI et al., 2015: PILLAY et al., 2015).

Nesse sentido, o objetivo desse estudo foi investigar a associação da presença dos polimorfismos rs182052 no gene ADIPOQ (Adiponectina), rs16835198 no gene FNDC5 (Fibronectin domain contain 5), rs6548238 no gene TMEM18 (Proteína transmembranar 18), e concentrações séricas de adiponectina e irisina com parâmetros bioquímicos e antropométricos em escolares de Santa Cruz do Sul/RS.

\section{FUNDAMENTAÇÃO TEÓRICA}

A obesidade tem origem multifatorial, decorrente de uma interação entre genética, fatores biológicos e ambientais (KUMAR et al., 2017). Diversos polimorfismos de nucleotídeo único têm se mostrado correlacionado com alterações bioquímicas e antropométricas (CODOÑER-FRACH et al., 2011; URSO et al., 2015).

O gene $A D I P O Q$ codifica uma proteína chamada adiponectina, que em baixos níveis está associada com acúmulo de gordura visceral, aumento de resistência à insulina e do risco de ter um primeiro evento cardiovascular (HAN et al., 2017; ZHANG et al., 2012). Por outro lado, altos níveis dessa proteína têm sido relacionados a um menor risco de desenvolver diabetes e problemas cardiovasculares (LINDBERG et al., 2015). No gene $A D I P O Q$, localizado na região 3q27.3 do cromossomo 3, está o SNP rs182052 que apresenta troca de alelos de A para G (NCBI, 2019). Estudos mostram a associação desse SNP com diabetes (WANG et al., 2015; $L i$ et al., 2015; BOSTROM et al., 2009). Kong et al. (2013), observaram que recém-nascidos com o alelo $G$ para esse SNP apresentavam maior peso ao nascer. Richardson et al. (2006), constataram uma relação entre o polimorfismo rs182052 e aumento do Índice de Massa Corporal (IMC).

Codificada pelo gene FNDC5, a irisina é uma proteína promissora como alvo terapêutico em doenças metabólicas (POLYZOS et al., 2018). Um estudo caso-controle realizado por Park et al. (2013), observaram que os níveis de irisina eram maiores em pacientes com síndrome metabólica do que o grupo controle. Hepatócitos humanos quando pré-tratados com irisina, demonstraram inibição da gliconeogênese e lipogênese pela ativação da via AMPK, uma importante rota reguladora do metabolismo de glicose e lipídeos (MO et al., 2016). 0 
polimorfismo rs16835198 no FNDC5, formado pela troca de alelos G/T, está localizado no cromossomo 1, na região 38.p12 (NCBI, 2019). Khidir et al. (2017) observaram uma relação entre o alelo G para esse SNP e um aumento de resistência à insulina e dislipidemia, enquanto o genótipo $\Pi$ T foi associado com uma diminuição do risco de desenvolver diabetes melitus do tipo 2

No cromossomo 2, região 2p25.3, está localizado o gene TMEM18, o qual apresenta o polimorfismo rs6548238, com troca de alelos T/C (NCBI, 2019). Um estudo conduzido por Rask-Andersen (2012), que utilizou como amostra 2.352 crianças gregas, constatou uma relação entre essa variante com circunferência da cintura e peso corporal. Esse polimorfismo também esteve associado com diabetes mellitus do tipo 2 após ajuste pelo IMC e aumento do risco de desenvolver obesidade (THOMSEN et al., 2012; ALMÈN et al., 2010). Portadores do alelo C para esse SNP demonstraram um aumento no IMC (PILLAY et al., 2015).

\section{MATERIAIS E MÉTODOS}

\subsection{DESENHO DO ESTUDO E AMOSTRA}

Estudo de caráter transversal, constituído por 500 crianças e adolescentes com idades de 7 a 17 anos, provenientes de escolas públicas e privadas da cidade de Santa Cruz do Sul/RS. São participantes da pesquisa intitulada como "SAÚDE DOS ESCOLARES - FASE III" aprovada pelo CEP da Universidade de Santa Cruz do Sul (UNISC) sob protocolo $n^{\circ} 714.216 / 14$. Nos anos de 2014 e 2015 foram realizadas as coletas das amostras e as mesmas armazenadas em freezer a $-80^{\circ} \mathrm{C}$. Os referenciais básicos da Bioética, previstos na Resolução 466/2012, foram atendidos pelo meio de assinatura, em duas vias, do Termo de Consentimento Livre e Esclarecido pelos pais ou responsáveis dos escolares convidados a participarem da pesquisa. Devido algumas variáveis não terem sido coletadas de todos os escolares, algumas categorias apresentam menos de 500 indivíduos.

\subsection{MENSURAÇÕES CLÍNICAS}

A coleta das amostras de sangue foi realizada após 12 horas de jejum. A partir de punção venosa foram coletados $10 \mathrm{~mL}$ de sangue. Dessa quantidade, $5 \mathrm{~mL}$ foram centrifugados para a separação do soro para a realização das análises bioquímicas. 0 s $5 \mathrm{~mL}$ restantes foram armazenadas em frascos com o anticoagulante ácido etilenodiamino tetra-acético (EDTA) para a extração de DNA pelo método de Miller et al. (1988). Os parâmetros bioquímicos analisados foram: Glicose (GLI), Colesterol Total (CT), Colesterol LDL (LDL-c), Colesterol HDL (HDL-c) e triglicerídeos (TAG). A equação de Friedwald et al. (1972) foi utilizada para calcular o LDL-c e as variáveis restantes foram determinadas a partir do equipamento Miura One (ISE, Roma, Itália). Utilizando como referência os dados do Instituto Nacional do Coração, Pulmão e Sangue (2011), foi considerado como ponte de corte para cada parâmetro: glicose $<100 \mathrm{mg} / \mathrm{dL}$ (normal) e risco $>100 \mathrm{mg} / \mathrm{dL}$, colesterol total $\geq 200 \mathrm{mg} / \mathrm{dL}$, c$\mathrm{LDL} \geq 130 \mathrm{mg} / \mathrm{dL}$, c-HDL <40mg/dL e triglicerídeos $\geq 100 \mathrm{mg} / \mathrm{dL}$ (0-9 anos) ou $\geq 130 \mathrm{mg} / \mathrm{dL}$ (10-19 anos).

0 índice de massa corporal (IMC,) circunferência da cintura (CC) e percentual de gordura (\%G) foram mensurados. O IMC foi classificado segundo a OMS (Organização Mundial da Saúde, 2007), sendo indivíduos eutróficos com o IMC entre $>18,50 \mathrm{~kg} / \mathrm{m}^{2}$ e $<24,99 \mathrm{~kg} / \mathrm{m}^{2}$ e risco $>25 \mathrm{~kg} / \mathrm{m}^{2}$. A circunferência da cintura foi mensurada através de uma fita métrica inelástica, sendo a parte mais estreita do tronco entre as costelas e a crista ilíaca e o quadril no nível do trocânter maior como referência para a medida. Posteriormente, os valores 
foram classificados de acordo com os critérios de Fernández et al. (2004), sendo CC normal <75 percentil e elevado com $\geq 90$ percentil.

A equação de Slaughter et al. (1988) foi utilizada para calcular o percentual de gordura, e após os dados foram classificados de acordo com os critérios de Lohman (1987), sendo separados em normal e risco conforme o sexo. Para meninos, o ponto de corte foi $20 \%$ de $\%$ G e para as meninas $25 \%$ de G\%, valores abaixo ou igual a esse referencial foram considerados como normal e acima como risco. As pressões arteriais sistólica e diastólica foram aferidas com os escolares sentados, após repouso, e foi utilizado o esfigmomanômetro junto com um estetoscópio no braço direito. Após foram classificadas como normal quando percentil <90 e risco quando percentil $\geq 90$ (SBC; SBH ; SBN, 2010).

\subsection{ANÁLISE MOLECULAR DOS GENES ADIPOQ, FNDC5 e TMEM18}

A partir do sangue total anticoagulado com EDTA, foi realizada a extração do DNA, através do método de Salting-out, descrito por Miller et al. (1988). Foram utilizados ensaios de discriminação alélica conforme as instruções do fabricante, os genes foram analisados pela técnica de qPCR no equipamento StepOne Plus. Os ensaios ADIPOQ rs182052 C__2412785_10, TMEM18 rs6548238 C_29311887_10, FNDC5 rs16835198 C_34204885_10 e Master Mix PCR Universal foram adquiridos da Applied Biosystem (Foster city, CA, USA). As análises foram realizadas no laboratório de Genética e Biotecnologia da UNISC.

\subsection{MENSURAÇÃO DA PROTEÍNA ADIPONECTINA E IRISINA}

Através do teste ELISA, a proteína adiponectina foi mensurada utilizando kit específico para a determinação quantitativa in vitro. 0 kit Adiponectin Human ELISA (Thermo Fisher Scientific, Waltham, MA, USA) foi utilizado seguindo as recomendações do fabricante.

A irisina foi mensurada através do kit Irisin, Recombinant (human, mouse, rat, canine) ELISA kit (Phoenix Pharmaceuticals, Burlingame, CA, USA). Para a realização do teste, foram seguidas as recomendações do fabricante.

\subsection{ANÁLISE E PROCESSAMENTO DE DADOS}

A partir do programa estatístico SPSS v.20, os dados foram tabulados e analisados. Através do teste chiquadrado, uma análise descritiva foi inicialmente realizada. 0 teste de Kolmogorov-Smirnov foi utilizado para determinar as amostras paramétricas e não-paramétricas. A equação de Hardy-Weinberg foi utilizada para calcular o equilíbrio dos polimorfismos analisados. 0 teste UNIANOVA ajustado por sexo e idade foi utilizado para analisar a relação entre os genótipos de cada SNP e concentrações sérica da adiponectina e irisina com IMC, CC, $\% G, P A D, P A S$, perfil lipídico e glicêmico.

\subsection{RESULTADOS E DISCUSSÃO}

A amostra do presente estudo está apresentada na Tabela 1. Foi composta predominantemente por escolares do sexo feminino (53,4\%). A maioria foram brancos $(69,94 \%)$, como o esperado, visto que a região tem descendência europeia (Filho e Monastério., 2012). Crianças com excesso de peso foram maioria (51,85\%), enquanto que apenas $32,40 \%$ dos adolescentes estavam nessa condição. 
Assim como previsto, em sua grande maioria (92,30\%), indivíduos com uma maior média para CC, estavam com excesso de peso. Patnaik et al. (2017) compararam a circunferência do pescoço (CP) e cintura entre indivíduos eutróficos e com sobrepeso ou obesidade. As medidas dessas variáveis foram relacionadas com - IMC. Nos resultados, foi observado que meninas com excesso de peso possuíam uma média maior para CC $(74,6 \pm 8,6 \mathrm{~cm})$ e CP $(31,1 \pm 2,5 \mathrm{~cm})$ quando comparadas com as medidas das eutróficas $(63,1 \pm 7,5 \mathrm{~cm}$; $28,2 \pm 2,2 \mathrm{~cm}$, respectivamente). Já para os meninos, esse dado se repete, àqueles com obesidade ou sobrepeso apresentavam médias superiores para CC $(80,5 \pm 12,7 \mathrm{~cm})$ e CP $(32,7 \pm 2,8)$ quando comparadas com os eutróficos que expressaram valores de $64,7 \pm 8,6 \mathrm{~cm}$ e $29 \pm 2,6$, respectivamente para essas variáveis. Dessa forma, o estudo sugere que medidas de CC e CP podem ser utilizadas na prática clínica e estudos epidemiológicos como um índice de sobrepeso/obesidade entre adolescentes em idade escolar (Patnaik et al., 2017).

Para as variáveis \%G, PAS e PAD, escolares com excesso de peso apresentaram médias significativamente maiores quando comparados com os eutróficos (Tabela 1). Também, nas categorias de risco desses parâmetros, indivíduos com sobrepeso ou obesidade representavam mais da metade. De acordo com os resultados encontrados, Hervás et al. (2015), de mesmo modo, observaram que valores da PAS e \%G corporal estavam significativamente elevados nos casos de excesso de peso e obesidade. Um estudo realizado em Belo Horizonte, com 1.450 estudantes, de 6 a 18 anos, observou que aqueles com sobrepeso ou obesidade tinham 2,7 vezes mais risco para ter uma pressão diastólica aumentada, quando comparados aos estudantes eutróficos (RIBEIRO et al., 2006).

Na categoria glicose, mais da metade $(61,12 \%)$ dos escolares classificados como risco, estavam com excesso de peso. Ainda, a média dessa variável estava maior nos indivíduos com IMC $>25 \mathrm{~kg} / \mathrm{m}^{2}(89 \pm 9 \mathrm{mg} / \mathrm{dL}$ $p=0,027)$ do que nos eutróficos $(87 \pm 7,34 \mathrm{mg} / \mathrm{dL})$. Romualdo et al. (2014) observaram resistência insulínica em crianças e adolescentes obesos, e que esta condição está associada com alterações clínicas e metabólicas.

Referente ao perfil lipídico, escolares com excesso de peso foram maioria na classificação de risco para os níveis séricos de LDL-c (53,15\%) e triglicerídeos (51,73\%), ainda, as médias dessas variáveis estavam elevadas nessa categoria quando comparadas com os eutróficos. A mediana de colesterol total nos indivíduos com excesso de peso (172 (151-191) $\mathrm{mg} / \mathrm{dL}, p=0,011)$ foi superior do que a encontrada nos eutróficos (164 (145-185) $\mathrm{mg} / \mathrm{dL}$ ). Skinner et al. (2015), também encontraram relação entre esses parâmetros bioquímicos com obesidade severa e sobrepeso. 0 estudo incluiu 8.579 crianças e jovens adultos, de idade entre 3 a 19 anos, participantes do NHANES (The National Center for Health Statistics). Nesse estudo, as categorias de peso foram consideradas como: sobrepeso ( $\geq 85$ a $<95$ percentil), obesidade classe I ( $\geq 95$ percentil a $<120 \%$ do percentil 95 ), obesidade classe II ( $\geq 120 \%$ a $<140 \%$ do percentil 95 , ou IMC $\geq 35$ ) e obesidade classe III ( $\geq 140 \%$ do percentil 95 , ou IMC $\geq 40$ ). Em participantes com sobrepeso, foi observada uma associação com colesterol $(p=<0,001)$, LDL-c $(p=0,02)$ e triglicerídeos $(p=<0,001)$. Porém, inversamente aos resultados obtidos, os autores observaram relação do HDL-c com excesso de peso.

Tabela 1 - Caracterização da amostra

\begin{tabular}{c|c|c|c}
\hline Variável & Eutrófico & Excesso de peso & Total \\
\hline Idade & $52(48,15)$ & $56(51,85)$ & 108 \\
Criança & $265(67,60)$ & $127(32,40)$ & 392 \\
Adolescente & & & \\
Sexo & $140(60,08)$ & $93(39,92)$ & 0,151 \\
Masculino & $177(66,30)$ & $90(33,70)$ & 233 \\
Feminino & $226(64,75))$ & $123(35,25)$ & 0,312 \\
Etnia & $90(60)$ & $60(40)$ & 150
\end{tabular}




\begin{tabular}{|c|c|c|c|c|}
\hline CC (cm) & $63,04( \pm 6,96)^{\star}$ & $73,24( \pm 12,23)^{*}$ & & $<0,001$ \\
\hline Normal & $310(75,80)$ & $99(24,20)$ & 409 & \\
\hline Risco & $7(7,70)$ & $84(92,30)$ & 91 & \\
\hline$\% G$ & $17,23(12,68-21,21)^{\star *}$ & $26,24(19,30-30,65)$ * * & & $<0,001$ \\
\hline Normal & $266(81,85)$ & $59(18,15)$ & 325 & \\
\hline Risco & $50(28,73)$ & $124(71,27)$ & 174 & \\
\hline PAS (mmHg) & $103,48( \pm 15,90)$ * & $108,85( \pm 16,82)^{*}$ & & 0,001 \\
\hline Normal & $273(66,58)$ & $137(33,42)$ & 410 & \\
\hline Risco & $43(48,31)$ & $46(51,69)$ & 89 & \\
\hline PAD (mmHg) & $63,83( \pm 11,20)^{*}$ & $67,29( \pm 16,82)^{*}$ & & 0,009 \\
\hline Normal & $278(65,72)$ & $145(34,28)$ & 423 & \\
\hline Risco & $38(50)$ & $38(50)$ & 76 & \\
\hline CT $(\mathrm{mg} / \mathrm{dL})$ & $164(145-185)$ * * & $172(151-191) * *$ & & 0,011 \\
\hline Normal & $187(68,50)$ & $86(31,50)$ & 273 & \\
\hline Risco & $238(71,47)$ & $95(28,53)$ & 333 & \\
\hline $\mathrm{GLI}(\mathrm{mg} / \mathrm{dL})$ & $87( \pm 7,34)^{*}$ & $89( \pm 9)^{*}$ & & 0,027 \\
\hline Normal & $308(64,43)$ & $170(35,57)$ & 478 & \\
\hline Risco & $7(38,88)$ & $11(61,12)$ & 18 & \\
\hline HDL-c (mg/dL) & $64,90(57,70-73,60) * *$ & $60,85(54,12-67,40)^{* *}$ & & 0,205 \\
\hline Normal & $310(64,05)$ & $174(35,95)$ & 484 & \\
\hline Risco & $5(45,45)$ & $6(54,55)$ & 11 & \\
\hline LDL-c (mg/dL) & $86,96(71,15-104,95) * *$ & $95,10(80,97-113,22) * *$ & & $<0,001$ \\
\hline Normal & $263(68,31)$ & $122(31,69)$ & 385 & \\
\hline Risco & $52(46,85)$ & $59(53,15)$ & 111 & \\
\hline $\mathrm{TAG}(\mathrm{mg} / \mathrm{dL})$ & $63,86( \pm 34,04)^{*}$ & $79,44( \pm 50,03) *$ & & $<0,001$ \\
\hline Normal & $259(68,15)$ & $121(31,85)$ & 380 & \\
\hline Risco & $56(48,27)$ & $60(51,73)$ & 116 & \\
\hline
\end{tabular}

Faixa Etária criança $<10$ anos, adolescente 10-17 anos. Índice de Massa Corporal (IMC): $>18,50 \mathrm{~kg} / \mathrm{m}^{2}$ e $<24,99$ $\mathrm{kg} / \mathrm{m}^{2}$ e risco $>25 \mathrm{~kg} / \mathrm{m}^{2}$. Circunferência da Cintura $(C C)$ : normal $(p<75)$ e risco $(p \geq)$ Percentual de Gordura (\%G): meninos: $<20 \%$ normal, risco $>20 \%$, meninas: normal $<25 \%$ e risco $>25 \%$. Pressão Sistólica e Diastólica: normal $(p<90)$, risco $(p \geq 90)$. Colesterol Total $(C T)$ : normal $<200 \mathrm{mg} / \mathrm{dL}$, risco $\geq 200 \mathrm{mg} / \mathrm{dL}$. Glicose (GLI): normal $<100$ $\mathrm{mg} / \mathrm{dL}$, risco $>100 \mathrm{mg} / \mathrm{dL}$. HDL-c: normal $>40 \mathrm{mg} / \mathrm{dL}$, risco $<40 \mathrm{mg} / \mathrm{dL}$. LDL-c: normal $<130 \mathrm{mg} / \mathrm{dL}$, risco $\geq$ $130 \mathrm{mg} / \mathrm{dL}$. Trigilicerídeos (TAG): normal $<100 \mathrm{mg} / \mathrm{dL}$, risco $\geq 100 \mathrm{mg} / \mathrm{dL}$. Valores expressos em média e desvio padrão*. * * Valores expressos em mediana e intervalo-interquartil. Valor significativo $(p<0,05)$.

De acordo com o que foi previamente descrito na literatura, todos os SNPs analisados estavam em equilíbrio de Hardy-Weinberg $p>0,05$ (JIANG et al., 2018; GARCìA-SOLís et al., 2016; TANG et al., 2014). 0 genótipo GG do $A D I P O Q$, apresentou a maior frequência (44,42\%), seguida pelo $A G(41,99 \%)$ e o alelo mais frequente foi o $\mathrm{G}(65,41 \%)$. Em relação ao TMEM18, o genótipo mais observado foi o CC (73,43\%), seguido pelo CT (23,93\%) e o alelo C foi o mais frequente (84,95\%). 0 genótipo GT foi o mais frequente para o FNDC5 (46,55\%), seguido por GG $(42,48 \%)$ e o alelo mais observado foi o $G(65,75 \%)$.

Tabela 2. Frequência genotípica e alélica dos escolares.

\begin{tabular}{|c|c|c|c|c|}
\hline Gene & $N$ & $\begin{array}{c}\text { Frequência } \\
\text { Observada }\end{array}$ & Frequência esperada & $p$ \\
\hline ADIPOQ & 493 & & & 0,270 \\
\hline$A A$ & & $67(13,59 \%)$ & 58 & \\
\hline$A G$ & & 207 (41,99\%) & 223 & \\
\hline GG & & $219(44,42 \%)$ & 215 & \\
\hline$A$ & & $341(34,58 \%)$ & & \\
\hline G & & $645(65,42 \%)$ & & \\
\hline TMEM18 & 493 & & & 0,895 \\
\hline$\pi$ & & $13(2,64 \%)$ & 11 & \\
\hline$C T$ & & $118(23,93 \%)$ & 123 & \\
\hline$C C$ & & $362(73,43 \%)$ & 359 & \\
\hline$T$ & & $149(15,05 \%)$ & & \\
\hline$C$ & & $841(84,95 \%)$ & & \\
\hline FNDC5 & 492 & & & 0,764 \\
\hline$T T$ & & $54(10,97 \%)$ & 58 & \\
\hline GT & & $229(46,55 \%)$ & 222 & \\
\hline GG & & $209(42,48 \%)$ & 212 & \\
\hline$T$ & & $337(34,25 \%)$ & & \\
\hline G & & $647(65,75 \%)$ & & \\
\hline
\end{tabular}


Os escolares que apresentavam excesso de portadores dos genótipos AA e AG para o SNP rs182052 no ADIPOQ, tiveram maior circunferência da cintura em comparação aos escolares eutróficos. Também, indivíduos com excesso de peso que portavam igualmente os genótipos AA e AG, tiveram uma maior média $(86,58 \pm 55,60 \mathrm{mg} / \mathrm{dL}, \mathrm{p}=0,021)$ para triglicerídeos quando comparados com indivíduos GG dessa mesma categoria $(69,56 \pm 39,37 \mathrm{mg} / \mathrm{dL})$ e aos escolares eutróficos AG+AA $(64,16 \pm 36,08 \mathrm{mg} / \mathrm{dL})$ e GG: $(63,98 \pm 31,75 \mathrm{mg} / \mathrm{dL}, \mathrm{p}=0,897)$ (Tabela 3).

Em contrapartida com os achados do estudo, Muñoz-Yánz et al. (2016), quando relacionaram variáveis antropométricas e bioquímicas, como circunferência da cintura e triglicerídeos, com o polimorfismo rs182052 no $A D I P O Q$, não houve significância estatística.

Tabela 3 - Comparação das médias dos dados antropométricos e bioquímicos com o SNP rs182052 no ADIPOQ.

\begin{tabular}{|c|c|c|c|c|c|c|c|c|}
\hline & \multicolumn{4}{|c|}{$\begin{array}{c}\text { EUTRÓFICOS } \\
\text { ADIPOQ rs182052 }\end{array}$} & \multicolumn{4}{|c|}{$\begin{array}{l}\text { EXCESSO DE PESO } \\
\text { ADIPOQ rs } 182052\end{array}$} \\
\hline & $\mathrm{n}$ & AG+AA & GG & $p$ & $\mathrm{n}$ & $A G+A A$ & GG & $p$ \\
\hline $\operatorname{IMC}\left(\mathrm{kg} / \mathrm{m}^{2}\right)$ & 314 & $\begin{array}{c}170 \\
(18,52 \pm 2,35)\end{array}$ & $\begin{array}{c}144 \\
(18,35 \pm 2,39)\end{array}$ & 0,291 & 187 & $\begin{array}{c}107 \\
24,21( \pm 4,60)\end{array}$ & $\begin{array}{c}76 \\
(23,90 \pm 3,76)\end{array}$ & 0,679 \\
\hline $\mathrm{CC}(\mathrm{cm})$ & 314 & $\begin{array}{c}170 \\
(63,12 \pm 6,94)\end{array}$ & $\begin{array}{c}144 \\
(63,14 \pm 6,89)\end{array}$ & 0,571 & 183 & $\begin{array}{c}107 \\
(74,55 \pm 13,54)\end{array}$ & $\begin{array}{c}76 \\
(71,39 \pm 10,15)\end{array}$ & 0,042 \\
\hline$\% G$ & 313 & $\begin{array}{c}169 \\
(17,26 \pm 5,74)\end{array}$ & $\begin{array}{c}144 \\
(17,48 \pm 5,69)\end{array}$ & 0,583 & 183 & $\begin{array}{c}107 \\
(25,29 \pm 8,02)\end{array}$ & $\begin{array}{c}76 \\
(25,49 \pm 8,13)\end{array}$ & 0,694 \\
\hline $\begin{array}{l}\text { PAD } \\
(\mathrm{mm} / \mathrm{Hg})\end{array}$ & 313 & $\begin{array}{c}169 \\
(64,04 \pm 11,59)\end{array}$ & $\begin{array}{c}144 \\
(63,79 \pm 10,75)\end{array}$ & 0,656 & 183 & $\begin{array}{c}107 \\
(68,11 \pm 11,81)\end{array}$ & $\begin{array}{c}76 \\
(66,13 \pm 11,59)\end{array}$ & 0,257 \\
\hline $\begin{array}{l}\text { PAS } \\
(\mathrm{mm} / \mathrm{Hg})\end{array}$ & 313 & $\begin{array}{c}169 \\
(103,56 \pm 15,7 \\
4)\end{array}$ & $\begin{array}{c}144 \\
(103,73 \pm 16,11)\end{array}$ & 0,801 & 183 & $\begin{array}{c}107 \\
(109,24 \pm 17,18)\end{array}$ & $\begin{array}{c}76 \\
(108,30 \pm 16,39)\end{array}$ & 0,297 \\
\hline CT (mg/dL) & 312 & $\begin{array}{c}169 \\
(163,60 \pm 28,5 \\
4)\end{array}$ & $\begin{array}{c}143 \\
(168,62 \pm 30,43)\end{array}$ & 0,121 & 181 & $\begin{array}{c}105 \\
(172,96 \pm 34,05)\end{array}$ & $\begin{array}{c}76 \\
(172,76 \pm 7,16)\end{array}$ & 0,948 \\
\hline GLI (mg/dL) & 312 & $\begin{array}{c}169 \\
(86,89 \pm 7,66)\end{array}$ & $\begin{array}{c}143 \\
(87,04 \pm 7,00)\end{array}$ & 0,985 & 181 & $\begin{array}{c}105 \\
(89,16 \pm 10,17)\end{array}$ & $\begin{array}{c}76 \\
(89,78 \pm 7,12)\end{array}$ & 0,783 \\
\hline $\begin{array}{l}\text { HDL-c } \\
(\mathrm{mg} / \mathrm{dL})\end{array}$ & 312 & $\begin{array}{c}169 \\
(65,10 \pm 10,62)\end{array}$ & $\begin{array}{c}143 \\
(65,77 \pm 12,52)\end{array}$ & 0,583 & 180 & $\begin{array}{c}104 \\
(59,43 \pm 10,79)\end{array}$ & $\begin{array}{c}76 \\
(61,94)\end{array}$ & 0,142 \\
\hline $\begin{array}{l}\text { LDL-c } \\
(\mathrm{mg} / \mathrm{dL})\end{array}$ & 312 & $\begin{array}{c}169 \\
(85,74 \pm 23,88)\end{array}$ & $\begin{array}{c}143 \\
(90,43 \pm 26,62)\end{array}$ & 0,091 & 181 & $\begin{array}{c}105 \\
(96,52 \pm 27,41)\end{array}$ & $\begin{array}{c}76 \\
(96,91 \pm 24,51)\end{array}$ & 0,910 \\
\hline $\mathrm{TAG}(\mathrm{mg} / \mathrm{dL})$ & 312 & $\begin{array}{c}169 \\
(64,16 \pm 36,08)\end{array}$ & $\begin{array}{c}143 \\
(63,98 \pm 31,75)\end{array}$ & 0,897 & 181 & $\begin{array}{c}105 \\
(86,58 \pm 55,60)\end{array}$ & $\begin{array}{c}76 \\
(69,56 \pm 39,37)\end{array}$ & 0,021 \\
\hline
\end{tabular}

UNIANOVA ajustado por sexo e idade. Valores expressos em média e desvio padrão. IMC: índice de massa corporal. CC: circunferência da cintura. \%G: percentual de gordura. PAD: pressão arterial diastólica. PAS: pressão arterial sistólica. CT: colesterol total. GLI: glicose. TAG: triglicerídeos.

Indivíduos eutróficos que portavam os genótipos GT+GG para o polimorfismo rs16835198 no FNDC5, apresentaram maiores médias para as variáveis IMC $(18,54 \pm 2,39, p=0,044)$ e circunferência da cintura $(63,39 \pm 6,85, p=0,033)$ quando comparados com os homozigotos $\Pi(18,54 \pm 2,39 ; 63,39 \pm 6,85$, respectivamente) dessa mesma categoria (Tabela 4).

Tang et al. (2014), realizaram um estudo caso-controle para investigar o efeito dos polimorfismos no FNDC5 na diabetes mellitus do tipo 2 e características clínicas relacionadas ao metabolismo da glicose. Diferentemente dos resultados obtidos, os autores não encontraram associação entre o SNP rs1683198 com o IMC, tanto nos grupos dos indivíduos com peso normal quantos nos com excesso de peso

De forma análoga aos resultados encontrados para CC, Todendi et al. (2018), também observaram uma relação entre a presença do polimorfismo rs1635198 com essa variável, sendo os portadores do genótipo TT com a maior média. 
Tabela 4 - Comparação das médias dos dados antropométricos e bioquímicos com o SNP rs16835198 no FNDC5.

\begin{tabular}{|c|c|c|c|c|c|c|c|c|}
\hline \multirow[b]{3}{*}{ IMC (kg/m²) } & \multicolumn{4}{|c|}{$\begin{array}{c}\text { EUTRÓFICO } \\
\text { FNDC5 rs16835198 }\end{array}$} & \multicolumn{4}{|c|}{$\begin{array}{l}\text { EXCESSO DE PESO } \\
\text { FNDC5 rs } 16835198\end{array}$} \\
\hline & $\mathrm{n}$ & GT+GG & $\pi$ & $p$ & $\mathrm{n}$ & $\mathrm{GT}+\mathrm{GG}$ & $\pi$ & $p$ \\
\hline & 313 & $\begin{array}{c}281 \\
(18,54 \pm 2,39)\end{array}$ & $\begin{array}{c}32 \\
(17,59 \pm 2,06)\end{array}$ & 0,044 & 179 & $\begin{array}{c}157 \\
(24,10 \pm 4,07)\end{array}$ & $\begin{array}{c}22 \\
(24,35 \pm 5,65)\end{array}$ & 0,301 \\
\hline $\mathrm{CC}(\mathrm{cm})$ & 313 & $\begin{array}{c}281 \\
(63,39 \pm 6,85)\end{array}$ & $\begin{array}{c}32 \\
(60,81 \pm 7,50)\end{array}$ & 0,033 & 179 & $\begin{array}{c}157 \\
(73,31 \pm 11,64)\end{array}$ & $\begin{array}{c}22 \\
(74,48 \pm 16,94)\end{array}$ & 0,137 \\
\hline$\% G$ & 312 & $\begin{array}{c}280 \\
(17,42 \pm 5,69)\end{array}$ & $\begin{array}{c}32 \\
(16,62 \pm 5,90)\end{array}$ & 0,976 & 179 & $\begin{array}{c}157 \\
(25,17 \pm 7,89)\end{array}$ & $\begin{array}{c}22 \\
(27,50 \pm 9,11)\end{array}$ & 0,071 \\
\hline $\mathrm{PAD}(\mathrm{mm} / \mathrm{Hg})$ & 312 & $\begin{array}{c}280 \\
(64,14 \pm 11,29)\end{array}$ & $\begin{array}{c}32 \\
(61,84 \pm 10,38)\end{array}$ & 0,377 & 179 & $\begin{array}{c}157 \\
(67,32 \pm 11,51)\end{array}$ & $\begin{array}{c}22 \\
(69,05 \pm 13,17)\end{array}$ & 0,243 \\
\hline PAS (mm/Hg) & 312 & $\begin{array}{c}280 \\
(104,29 \pm 16,08)\end{array}$ & $\begin{array}{c}32 \\
(98,38 \pm 13,21)\end{array}$ & 0,055 & 179 & $\begin{array}{c}157 \\
(108,76 \pm 16,53)\end{array}$ & $\begin{array}{c}22 \\
(110,82 \pm 19,99)\end{array}$ & 0,133 \\
\hline CT (mg/dL) & 311 & $\begin{array}{c}279 \\
(165,41 \pm 29,73)\end{array}$ & $\begin{array}{c}32 \\
(170,34 \pm 27,74)\end{array}$ & 0,363 & 177 & $\begin{array}{c}155 \\
(172,58 \pm 32,00)\end{array}$ & $\begin{array}{c}22 \\
(176,31 \pm 27,30)\end{array}$ & 0,389 \\
\hline $\mathrm{GLI}(\mathrm{mg} / \mathrm{dL})$ & 311 & $\begin{array}{c}279 \\
(87,04 \pm 7,11)\end{array}$ & $\begin{array}{c}32 \\
(86,12 \pm 9,38)\end{array}$ & 0,381 & 177 & $\begin{array}{c}155 \\
(89,84 \pm 9,06)\end{array}$ & $\begin{array}{c}22 \\
(85,86 \pm 8,54)\end{array}$ & 0,150 \\
\hline HDL-c (mg/dL) & 311 & $\begin{array}{c}279 \\
(65,03 \pm 11,44)\end{array}$ & $\begin{array}{c}32 \\
(69,00 \pm 11,74)\end{array}$ & 0,110 & 176 & $\begin{array}{c}154 \\
(60,32 \pm 10,78)\end{array}$ & $\begin{array}{c}22 \\
(59,44 \pm 9,79)\end{array}$ & 0,654 \\
\hline LDL-c (mg/dL) & 311 & $\begin{array}{c}279 \\
(87,74 \pm 25,12)\end{array}$ & $\begin{array}{c}32 \\
(89,17 \pm 26,97)\end{array}$ & 0,685 & 177 & $\begin{array}{c}155 \\
(96,45 \pm 26,56)\end{array}$ & $\begin{array}{c}22 \\
(100,48 \pm 23,27)\end{array}$ & 0,340 \\
\hline TAG (mg/dL) & 311 & $\begin{array}{c}279 \\
(64,93 \pm 34,93)\end{array}$ & $\begin{array}{c}32 \\
(56,22 \pm 25,66)\end{array}$ & 0,255 & 177 & $\begin{array}{c}155 \\
(80,16 \pm 50,58)\end{array}$ & $\begin{array}{c}22 \\
(81,92 \pm 48,30)\end{array}$ & 0,550 \\
\hline
\end{tabular}

UNIANOVA ajustado por sexo e idade. Valores expressos em média e desvio padrão. IMC: índice de massa corporal. CC: circunferência da cintura. \%G: percentual de gordura. PAD: pressão arterial diastólica. PAS: pressão arterial sistólica. CT: colesterol total. GLI: glicose. TAG: triglicerídeos.

Escolares com sobrepeso ou obesidade portadores do genótipo CC para o polimorfismo rs6548238 no TMEM18 apresentaram maior média $(175,61 \pm 31,88 \mathrm{mg} / \mathrm{dL}, p=0,021)$ de colesterol total quando comparados com aqueles que possuíam CT ou $\Pi(162,31 \pm 27,93 \mathrm{mg} / \mathrm{dL})$. Também, a média dessa variável se manteve superior quando foi relacionada com indivíduos eutróficos heterozigotos e homozigotos (CT+TT: $163,82 \pm 29,32 \mathrm{mg} / \mathrm{dL}$ e CC: $166,75 \pm 29,57 \mathrm{mg} / \mathrm{dL} p=0,393$ ) (Tabela 5).

Para o estudo, foram considerados estatisticamente significativos valores de $p<0,05$. Porém, foi observado que a variável LDL-c, mesmo tendo ficado levemente acima do valor de significância $(p=0,05)$, apresentou níveis séricos elevados em indivíduos com excesso de peso para o genótipo CC. Todendi et al. (2018), realizaram um estudo transversal com 1471 crianças e adolescentes, de idades entre 6 e 17 anos, de escolas de Santa Cruz do Sul. Ao investigar o efeito do polimorfismo rs6548238 no TMEM18, com características antropométricas e parâmetros metabólicos, não encontraram relação estatística com a variável LDL-c.

Tabela 5 - Comparação das médias dos dados antropométricos e bioquímicos com o SNP rs6548238 no TMEM18

\begin{tabular}{|c|c|c|c|c|c|c|c|c|}
\hline & \multicolumn{4}{|c|}{$\begin{array}{c}\text { EUTRÓFICOS } \\
\text { TMEM18 rs6548238 }\end{array}$} & \multicolumn{4}{|c|}{$\begin{array}{l}\text { EXCESSO DE PESO } \\
\text { TMEM18 rs } 6548238\end{array}$} \\
\hline & $\mathrm{n}$ & $\mathrm{CT}+\mathrm{TT}$ & $\mathrm{CC}$ & $p$ & $\mathrm{n}$ & $\mathrm{CT}+\mathrm{TT}$ & $\mathrm{CC}$ & $p$ \\
\hline $\operatorname{IMC}\left(\mathrm{kg} / \mathrm{m}^{2}\right)$ & 314 & $\begin{array}{c}90 \\
(18,41 \pm 2,37)\end{array}$ & $\begin{array}{c}224 \\
(18,45 \pm 2,29)\end{array}$ & 0,928 & 179 & $\begin{array}{r}41 \\
(23,5 \pm 3,85)\end{array}$ & $138(24,28 \pm 4,40)$ & 0,422 \\
\hline $\mathrm{CC}(\mathrm{cm})$ & 314 & $\begin{array}{c}90 \\
(62,75 \pm 7,38)\end{array}$ & $\begin{array}{c}224 \\
(63,6,76)\end{array}$ & 0,911 & 179 & $\begin{array}{c}41 \\
(73,03 \pm 11,24)\end{array}$ & $\begin{array}{c}138 \\
(73,31 \pm 12,69)\end{array}$ & 0,869 \\
\hline$\% G$ & 313 & $\begin{array}{c}90 \\
(17,46 \pm 5,41)\end{array}$ & $\begin{array}{c}223 \\
(17,32 \pm 5,84)\end{array}$ & 0,679 & 179 & $\begin{array}{c}41 \\
(25,16 \pm 8,05)\end{array}$ & $\begin{array}{c}138 \\
(25,44 \pm 8,02)\end{array}$ & 0,921 \\
\hline PAD (mm/Hg) & 313 & $\begin{array}{c}90 \\
(64,98 \pm 12,30)\end{array}$ & $\begin{array}{c}223 \\
(63,50 \pm 10,72)\end{array}$ & 0,214 & 179 & $\begin{array}{c}41 \\
(66,27 \pm 10,63)\end{array}$ & $\begin{array}{c}138 \\
(67,62 \pm 11,95)\end{array}$ & 0,637 \\
\hline PAS (mm/Hg) & 313 & $\begin{array}{c}90 \\
(104,21 \pm 16,40)\end{array}$ & $\begin{array}{c}223 \\
(103,41)\end{array}$ & 0,462 & 179 & $\begin{array}{c}41 \\
(108,10 \pm 14,58)\end{array}$ & $\begin{array}{c}138 \\
(109,04 \pm 17,53)\end{array}$ & 0,897 \\
\hline CT (mg/dL) & 312 & $\begin{array}{c}90 \\
(163,82 \pm 29,32)\end{array}$ & $\begin{array}{c}222 \\
(166,75 \pm 29,57)\end{array}$ & 0,393 & 177 & $\begin{array}{c}41 \\
(162,31 \pm 27,93)\end{array}$ & $\begin{array}{c}136 \\
(175,61 \pm 31,88)\end{array}$ & 0,021 \\
\hline
\end{tabular}




\begin{tabular}{|c|c|c|c|c|c|c|c|c|}
\hline GLI (mg/dL) & 312 & $\begin{array}{c}90 \\
(85,73 \pm 8,14)\end{array}$ & $\begin{array}{c}222 \\
(87,73)\end{array}$ & 0,103 & 177 & $\begin{array}{c}41 \\
(89,29 \pm 8,15)\end{array}$ & $\begin{array}{c}136 \\
(89,47 \pm 9,39)\end{array}$ & 0,939 \\
\hline HDL-c (mg/dL) & 312 & $\begin{array}{c}90 \\
(64,27 \pm 10,94)\end{array}$ & $\begin{array}{c}222 \\
(65,87 \pm 11,73)\end{array}$ & 0,326 & 176 & $\begin{array}{c}41 \\
(58,80 \pm 8,89)\end{array}$ & $\begin{array}{c}135 \\
(60,74)\end{array}$ & 0,272 \\
\hline LDL-c (mg/dL) & 312 & $\begin{array}{c}90 \\
(87,13 \pm 26,33)\end{array}$ & $\begin{array}{c}222 \\
(88,20 \pm 24,84)\end{array}$ & 0,654 & 177 & $\begin{array}{c}41 \\
(89,09 \pm 24,48)\end{array}$ & $\begin{array}{c}136 \\
(98,66 \pm 26,58)\end{array}$ & ,050 \\
\hline $\mathrm{TAG}(\mathrm{mg} / \mathrm{dL})$ & 312 & $\begin{array}{c}90 \\
(60,05 \pm 25,31)\end{array}$ & $\begin{array}{c}222 \\
(65,72 \pm 37,02)\end{array}$ & 0,149 & 177 & $\begin{array}{c}41 \\
(72,17 \pm 36,70)\end{array}$ & $\begin{array}{c}136 \\
(82,21 \pm 53,58)\end{array}$ & 323 \\
\hline
\end{tabular}

UNIANOVA ajustado por sexo e idade. Valores expressos em média e desvio padrão. IMC: índice de massa corporal. CC: circunferência da cintura. \%G: percentual de gordura. PAD: pressão arterial diastólica. PAS: pressão arterial sistólica.CT: colesterol total. GLI: glicose. TAG: triglicerídeos.

Não foi observada relação estatística significativa entre os níveis séricos da adiponectina e irisina com alterações em parâmetros antropométricos e bioquímicos dos escolares estudados.

\section{CONSIDERAÇÕES FINAIS}

Os escolares com excesso de peso representavam mais da metade na classificação de riscos das variáveis idade, circunferência da cintura, percentual de gordura, pressão arterial sistólica e diastólica, LDL-c e triglicerídeos. Portadores dos genótipos AG+AA para o SNP rs182052 no ADIPOQ demonstraram maiores médias de circunferência da cintura e triglicerídeos. Escolares eutróficos com os genótipos GT e GG para o polimorfismo rs16835198 no FNDC5 foram associados a maiores médias para IMC e circunferência da cintura quando comparados com os portadores do genótipo TT. Indivíduos com o genótipo GG para o polimorfismo rs6538238 no TMEM18 demonstraram associação com maiores níveis de colesterol. Níveis séricos de adiponectina e irisina, não demonstraram associação com os parâmetros estudados.

\section{REFERÊNCIAS}

ALMÉN, MS. et al. The obesity gene, TMEM18, is of ancient origin, found in majority of neuronal cells in all major brain regions and associated with obesity in severely obese children. BMC Medical Genetics, v.11, n.58, 2010.

ARROYO-JOHNSON C, MINCEY K.D. Obesity Epidemiology Worldwide. Gastroenterol Clinics of North America, v.45, n.4, p.571-579, 2016.

BOSTROM, P. et al. A PGC1- $\alpha$-dependent myokine that drives brown-fat-like development of white fat and thermogenesis. Nature, v. 481, n.7382, p.463-8, 2012,

FERNÁNDEZ, J. R. et al. Waist circumference percentiles in nationally representative samples of African-American, European-American, and Mexican-American children and adolescents. The Journal of Pediatrics, Cincinnati, v. 145, n. 4, p. 439-444, 2004.

FILHO I.C, MONASTÉRIO L. Immigration and the origins of regional inequality: Government-sponsored European migration to southern Brazil before World War I. Regional Science and Urban Economics. v. 42, n.5, p.794-807, 2012.

FRIEDEWALD, W. T. et al. Estimation of the concentration of low-density lipoprotein cholesterol in plasma, without use of the preparative ultracentrifuge. Clinical Chemisty, Baltimore, v. 18, n. 6, p. 499502, 1972.

GÁRCIA-SOLís, P. et al. Fat mass obesity-associated (FTO) (rs9939609) and melanocortin 4 receptor (MC4R) (rs17782313) SNP are positively associated with obesity and blood pressure in Mexican school-aged children. British Journal of nutrition, v. 116, n.10, p. 1834-1840, 2016.

HAN, S.J. et al. Low Plasma Adiponectin Concentrations Predict Increases in Visceral Adiposity and Insulin Resistance. Journal of Clinical Endocrinology and Metabolism, v.102, n.12, p.4626-4633, 2017.

HERVÁS A. I. G. et al. Pression sistólica, obesidade abdominal y grasa corporal predictores del sídrome metabólico em pré-escolares españoles. Nutrición Hospitalaria, v.31, n.5, p. 2109-2114, 2015. 
Instituto Nacional do Coração, Pulmão e Sangue. NHLBI. Painel de especialistas em diretrizes integradas para saúde cardiovascular e redução de risco em crianças e adolescentes. Bethesda: 2012.

JAAKKOLA, J.M., et al. Association of Adiponectin with Adolescent Cardiovascular Health in a Dietary Intervention Study. The Journal of Pedriatics. v.167, n.2, p. 353-60, 2015.

JIANG, L. et al. Obesity, osteoarthritis and genetic risk: The rs182052 polymorphism in the ADIPOQ gene is potentially associated with risk of knee osteoarthritis. Bone Joint Research, v.7, n.7, p:494500, 2018.

KHIDIR, E.G., et al. Association of irisin and FNDC5 rs16835198 G > T gene polymorphism with type 2 diabetes mellitus and diabetic nephropathy. An Egyptian pilot study. Gene. v.626, p.26-31, 2017

KINLEN, D. et al. Complications of obesity. QJM: An International Journal of Medicine, v.111, n.7, p.437-443, 2017.

KONG, K. A. et al. Association of Adiponectin Gene Polymorphism With Birth Weight in Korean Neonates. Twin Research and Human Genetics, Cambridge. v.16, n.03, p. 732-738, 2013.

KUMAR, S., e KELLY, A. S. Review of Childhood Obesity. Mayo Clinic Proceedings, v.92, n.2, p. 251-65, 2017.

KYROU, L. et al. Adiponectin circulating levels and 10-year (2002-2012) cardiovascular disease incidence: the

ATTICA Study. Endocrine, v.58, n.3, p.542-552, 2017.

LEÓN-MIMILA, P. et al. Contribution of common genetic variants to obesity and obesity-related traits in mexican children and adults. PLoS One, v.8, n.8, 2013.

LI, Z-P. et al. Relation between ADIPOQ Gene Polymorphisms and Type 2 Diabetes. Genes v. 6, n.3, p. 512-9. 8, 2015.

LINDBERG, S. et al Adiponectin, type 2 diabetes and cardiovascular risk. European Journal of Preventive Cardiology, v.22, n.3, p. 276-283, 2013.

LOHMAN, T. The use of skinfold to estimate body fatness on children and youth. Journal of Physical Education, Recreation e Dance, London, v. 58, n. 9, p. 98-102, 1987.

MO L. et al. Irisin Is Regulated by CAR in Liver and Is a Mediator of Hepatic Glucose and Lipid Metabolism. Molecular Endocrinology, v.30, n.5, p.533-42, 2016.

NATIONAL CENTER FOR BIOTECHNOLOGY INFORMATION, NCBI. Disponível em: https://www.ncbi.nlm.nih.gov/snp/rs182052. Acesso dia: 17/03/2019.

NATIONAL CENTER FOR BIOTECHNOLOGY INFORMATION, NCBI. Disponível em: https://www.ncbi.nlm.nih.gov/snp/rs6548238. Acesso dia: 17/03/2019.

NATIONAL CENTER FOR BIOTECHNOLOGY INFORMATION, NCBI. Disponível em: https://www.ncbi.nIm.nih.gov/snp/rs16835198. Acesso dia: 17/03/2019.

NCD Risk Factor Collaboration (NCD-RisC). Worldwide trends in body-mass index, underweight, overweight, and obesity from 1975 to 2016: a pooled analysis of 2416 population-based measurement studies in 128.9 million children, adolescents, and adults. Lancet, v.390, n.10113, p.2627-2642, 2017.

ONIS M, et al. Development of a WHO growth reference for school-aged children and adolescents. Bull World Health Organ. v.5, n.9, p.660-667, 2007.

ORGANIZAÇÃO MUNDIAL DA SAÚDE. Classificação do Índice de Massa Corporal. Disponível em: http://apps.who.int/bmi/index.jsp?introPage=intro_3.html. Acesso dia: 17/03/2019.

PARK, K.H. et al. Circulating irisin in relation to insulin resistance and the metabolic syndrome. Journal of Clinical Endocrinology and Metabolism, v.98, n.12, p.4899-907, 2013.

PATNAIK, L. et al. Validating Neck Circumference and Waist Circumference as Anthropometric Measures of Overweight/Obesity in Adolescents. Indian Pediatris, Nova Déli. v.54, n.5, p.377-380, 2017. 
PILLAY, V. et al. Exploring genetic markers of adult obesity risk in black adolescent South Africans-the Birth to Twenty Cohort. Nutrition \& Diabetes. Londres. v.5, n.6, p.157-, 2015.

POLYZOS, S. A. et al. Irisin in metabolic diseases. Endocrine, v.59, n.2, p.260-274, 2017.

RASK-ANDERSEN, M. et al., Association of TMEM18 variants with BMI and waist circumference in children and correlation of mRNA expression in the PFC with body weight in rats. European Journal of Human Genetics, v. 20, n.2, p.192-197, 2012.

RIBEIRO, R. Q. C. et al. Fatores adicionais de risco cardiovascular associados ao excesso de peso em crianças e adolescentes: o estudo do coração de Belo Horizonte. Arquivos Brasileiros de Cardiologia. vol.86, n.6, 2006.

RICHARDSON, D. K. et al. Association between variants in the genes for adiponectin and its receptors with insulin resistance syndrome (IRS)-related phenotypes in Mexican Americans. Diabetologia, Bristol.v.49, n.10, p. 23172328, 2006.

ROMUALDO, M. C. dos S., et al. Insulin resistance in obese children and adolescents. Jornal de Pediatria, v. 9, n.6, p.600-607, 2014.

SKINNER, A.C. et al. Cardiometabolic Risks and Severity of Obesity in Children and Young Adults. New England Journal of Medicine, Waltham. v. 373, n. 14, p.1307-17, 2015.

SLAUGTHER, M. H. et al. Skinfold equations for estimation of body fatness in children and youth. Human Biology, Washington, v. 60, n. 5, p. 709-723, 1988.

SOCIEDADE BRASILEIRA DE CARDIOLOGIA (SBC), SOCIEDADE BRASILEIRA DE HIPERTENSÃO (SBH), SOCIEDADE BRASILEIRA DE NEFROLOGIA (SBN). VI Diretrizes Brasileiras de Hipertensão. Arq Bras Cardiol., São Paulo, v. 95, n. 1 supl.1, p. 1-51, 2010.

TANG, S. et al. An interaction between a FNDC5 variant and obesity modulates glucose metabolism in a Chinese Han population. PLoS One, v.9, n.11, 2014.

THOMSEN, M. et al. ß2-Adrenergic Receptor Thr164lle Polymorphism, Obesity, and Diabetes: Comparison with FTO, MC4R, andTMEM18Polymorphisms in More Than 64,000 Individuals. The Journal of Clinical Endocrinology \& Metabolism, Washington. v.97, n.6, p.1074-79, 2012.

TODENDI, P.F. et al. Genetic risk score based on fat mass and obesity-associated, transmembrane protein 18 and fibronectin type III domain containing 5 polymorphisms is associated with anthropometric characteristics in South Brazilian children and adolescents. British Journal of Nutrition, Cambridge. v.121, n.1, p.93-9, 2019.

WANG, W.L. et al. Relation between ADIPOQ gene polymorphisms and type 2 diabetes in a Chinese population. International Journal of Clinical and Experimental Medicine, v.8, n.4, p. 6124-28, 2015.

YADAV, A. et al. Role of leptin and adiponectin in insulin resistance. Clinica Chimica Acta, v.417, p.80-84. 2013.

ZHANG, B.-C., Liu, W.-J., Che, W.-L., \& Xu, Y.-W. (2012). Serum total adiponectin level and risk of cardiovascular disease in Han Chinese populations: a meta-analysis of 17 case-control studies. Clinical Endocrinology, v.77, n.3, p. 370-378, 2012 